\title{
Preoperative SPECT imaging as a tool for surgical planning in patients with axial neck and back pain
}

\author{
G. Damian Brusko, BS, ${ }^{1}$ Roberto J. Perez-Roman, MD, ${ }^{1}$ Harold Tapamo, BS, ${ }^{1}$ S. Shelby Burks, MD, ${ }^{1}$ \\ Aldo N. Serafini, MD, ${ }^{2}$ and Michael Y. Wang, MD'
}

'Department of Neurological Surgery and 2Division of Nuclear Medicine, Department of Radiology, University of Miami Miller
School of Medicine, Miami, Florida

OBJECTIVE Hybrid SPECT with CT imaging has been used to help elucidate pain generators in patients with axial neck and back pain, identifying potential sites for treatment. Few studies have examined its role in spine surgery and most literature focuses on its use postoperatively. The authors describe the largest series to date of patients with symptomatic spondylosis who underwent preoperative SPECT imaging for surgical planning.

METHODS A retrospective medical and imaging record review was conducted to identify patients who underwent SPECT or SPECT/CT studies between January 2014 and May 2018. Patients who underwent spine surgical intervention for spondylosis with primary symptoms of axial neck or back pain and who had evidence of hypermetabolic foci on spinal SPECT imaging were included. Only those patients who subsequently underwent surgery on a spinal level associated with increased radiotracer uptake were included in the analysis. Patient baseline and demographic information, and data pertaining to SPECT imaging, surgical planning, and postoperative care were collected and analyzed.

RESULTS A total of 23 patients with an average age at surgery of $60.0 \pm 11.0$ years were included. Fifteen patients $(65.2 \%)$ were male. A total of 53 spinal levels were treated, with an average of 2.30 levels treated per patient. All patients underwent fusion surgery, either lumbar $(n=14)$, with interbody fusion most commonly used $(64.2 \%)$; or cervical $(n=$ 9 ), with anterior cervical discectomy and fusion (66.6\%) being the most common. The average length of hospital stay was $3.45 \pm 2.32$ days. One patient developed a wound infection postoperatively, requiring readmission. At the 3-month follow-up, 18 patients (78.3\%) reported clinical improvement in pain. Eleven patients (47.8\%) reported complete symptom resolution at the 6-month follow-up. At 1 year postoperatively, 19 patients $(82.6 \%)$ reported significant relief of their symptoms following surgery.

CONCLUSIONS This is the largest series to date describing patients with axial neck and back pain who underwent preoperative SPECT imaging and subsequent surgical intervention on the affected spinal levels. The results demonstrate that SPECT imaging may be a useful adjunct to guide surgical planning, resulting in substantial clinical improvement following surgery. https://thejns.org/doi/abs/10.3171/2019.9.FOCUS19648

KEYWORDS axial pain; imaging; preoperative planning; single-photon emission computed tomography; SPECT; spine surgery

$\mathrm{H}$ IGH-RESOLUTION SPECT or hybrid SPECT/CT imaging has been increasingly used as a spinal imaging modality. When other imaging studies appear inconclusive, SPECT can be used to highlight sites of mechanical stress and degeneration, especially when combined with CT. ${ }^{9}$ However, use of SPECT imaging in the perioperative period has not been well described in the existing literature.

Few studies have examined SPECT or hybrid SPECT/ $\mathrm{CT}$ imaging to evaluate persistent or recurrent pain fol- lowing spine surgery when conventional imaging modalities such as CT or MRI were inconclusive. ${ }^{2,3}$ However, the utility of postoperative SPECT imaging, particularly in the early postoperative period, is limited because increased osteoblastic activity related to bony fusion results in a high degree of radiotracer uptake on SPECT imaging.

Thus, a more clinically impactful use for SPECT imaging may be during the preoperative period. SPECT and hybrid SPECT/CT imaging have recently aided identification of pain generators in patients with axial neck and 
back pain, indicating potential sites of degeneration. ${ }^{13,14}$ Primarily occurring in the facet joints, the incidence of arthropathy identified on SPECT imaging ranges between $42 \%$ and $48 \% .{ }^{8}$ Despite the common occurrence of facet hypermetabolism, there is a paucity of literature describing SPECT imaging as a preoperative planning tool. In the present study, we describe the largest series to date of patients with axial neck or back pain who underwent preoperative SPECT imaging for surgical planning. We also examine the effectiveness of surgical intervention based on patient-reported improvements during a 1-year followup period.

\section{Methods \\ Patient Population}

A retrospective medical and imaging record review was conducted to identify all adult patients (age $>18$ years) who underwent SPECT or SPECT/CT studies between January 2010 and April 2018. Patients who presented with primary symptoms of axial neck or back pain and who had evidence of hypermetabolic foci on spine SPECT imaging were identified. In addition to a primary complaint of axial pain, the majority of patients had concomitant diagnoses of spinal stenosis, mild spondylolisthesis, spondylosis, or a spinal deformity such as scoliosis or kyphosis. Two patients preoperatively reported symptoms of a radiculopathy in addition to axial neck and back pain. All patients with a diagnosed neoplasm, infections, inflammatory spondyloarthropathy, history of trauma, or any pathological fracture were excluded. Demographic and baseline characteristics were collected for each patient, and radiographic reports and images for SPECT scans were also reviewed. Clinical information regarding surgical intervention, in-hospital postoperative care, and clinic visits at 3,6 , and 12 months were collected as well. Provider clinic notes from follow-up visits were examined to determine the extent of axial pain resolution during each visit based on the patient's subjective reporting of their symptomatology. Patients were then grouped into one of 3 categories: "no improvement or worsening," "some improvement," or "complete resolution."

\section{SPECT Imaging}

High-resolution SPECT imaging was completed using a standardized spine-bone protocol. Following 24-mCi intravenous injections of ${ }^{99} \mathrm{Tc}-$ methylene diphosphonate, a triple-phase planar bone scintigraphy with immediate and delayed images, whole-body bone scans, and tomographic imaging using SPECT or SPECT/CT was performed (ADAC Vertex dual head scanner; Philips Medical Systems). Patients were identified based on the Current Procedural Terminology (CPT) codes 78315, 78320, or 78306. Triple phase, tomographic SPECT, and whole-body bone and/or joint imaging procedures were included for review, and hybrid SPECT/CT or plain SPECT protocols were included. A nuclear medicine radiologist evaluated all imaging studies, characterizing increased radionuclide uptake as hypermetabolic foci secondary to active or increased osteoblastic activity. The number, level, and laterality of increased radionuclide uptake in the cervical, thoracic, and lumbar spine were recorded for each patient based on a review of the imaging report.

\section{Surgical Interventions}

Following the identification of hypermetabolism on SPECT imaging, a subset of included patients underwent surgical intervention. Selection of patients for surgery was augmented by preoperative SPECT imaging when other conventional imaging modalities such as radiography, CT, and MRI failed to identify a clear pain generator or in patients with multilevel spinal pathologies as potential culprits for axial pain. All available imaging studies, clinical presentation, and physical examination findings as well as surgeon and patient preferences were used in the surgical decision-making process. Only those patients who subsequently underwent spine surgery with the senior author (M.Y.W.) on a spinal level associated with increased radiotracer uptake were included in the final analysis. All patients underwent a spinal fusion procedure, with the specific technique determined by clinical indications and surgeon preference.

\section{Statistical Analysis}

Categorical variables are reported with percentages and continuous variables are reported as means with standard deviation. Data analysis was conducted using Excel 2016 (Microsoft Corp.).

\section{Results}

\section{Baseline and Radiographic Characteristics}

A total of 23 patients underwent spine surgery on a hypermetabolic level following identification on SPECT imaging. The average age at time of surgery was $60.0 \pm 11.0$ years; 15 patients $(65.2 \%)$ were male and 8 were female. Twenty patients (86.9\%) underwent hybrid SPECT/CT imaging. The primary indications for SPECT imaging were pseudarthrosis (37.5\%), axial back pain (32.0\%), and axial neck pain $(28.1 \%)$. Fifteen patients $(65.2 \%)$ demonstrated evidence of degenerative pathology in the lumbar spine, 9 patients (39.1\%) in the cervical spine, and 5 patients (21.7\%) in the thoracic spine. Five patients had involvement of more than one spinal region. The incidence of increased radionuclide uptake per spinal level is shown in Table 1.

\section{Surgical Intervention and Outcomes}

A total of 53 spinal levels were treated, with an aver-

TABLE 1. Number of patients with SPECT hypermetabolism per spinal level

\begin{tabular}{|c|c|c|c|c|c|}
\hline \multicolumn{2}{|c|}{ Cervical } & \multicolumn{2}{|c|}{ Thoracic } & \multicolumn{2}{|c|}{ Lumbar } \\
\hline Level & No. & Level & No. & Level & No. \\
\hline C2-3 & 2 & T5-6 & 1 & L1-2 & 1 \\
\hline C3-4 & 4 & T10-11 & 1 & L2-3 & 7 \\
\hline C4-5 & 2 & T12-L1 & 2 & L3-4 & 7 \\
\hline C5-6 & 2 & & & L4-5 & 5 \\
\hline C6-7 & 1 & & & L5-S1 & 6 \\
\hline
\end{tabular}


TABLE 2. Frequency of surgical interventions following preoperative SPECT imaging

\begin{tabular}{ll}
\hline \multicolumn{1}{c}{ Procedure } & No. \\
\hline Cervical & 6 \\
\hline Anterior cervical discectomy and fusion (ACDF) & 3 \\
\hline Anterior corpectomy w/ fusion & 3 \\
\hline Lumbar & 3 \\
\hline Anterior lumbar interbody fusion (ALIF) & 1 \\
\hline Lateral lumbar interbody fusion (LLIF) & 1 \\
\hline Transforaminal lumbar interbody fusion (TLIF) & 5 \\
\hline Posterior lumbar interbody fusion (PLIF) & 1 \\
\hline Posterior spinal fusion w/ instrumentation & \\
\hline Posterolateral instrumentation and fusion &
\end{tabular}

age of 2.30 levels treated per patient. All patients underwent fusion surgery. In patients with lumbar pathology ( $\mathrm{n}=14$ ), lumbar interbody fusion was most commonly used (57.1\%). Patients with evidence of hypermetabolism on cervical SPECT imaging $(\mathrm{n}=9)$ underwent anterior cervical discectomy and fusion (66.6\%) most commonly (Table 2). Other common surgical procedures included posterior instrumented fusion and cervical corpectomy with fusion. The average length of hospital stay was 3.45 \pm 2.32 days. One patient developed a wound infection postoperatively, requiring readmission and treatment 1 month after surgery. There were no intraoperative complications. At the 3-month follow-up visit, 18 patients (78.3\%) reported clinical improvement in pain, and at 6 months, 11 patients $(47.8 \%)$ reported complete symptom resolution. Nineteen patients $(82.6 \%)$ reported improvement in their symptoms 1 year following surgery. No patients required reoperation during the follow-up period, nor did any patients experience acute worsening of their symptoms following surgery. Case examples illustrating patients who underwent cervical (Fig. 1) and lumbar (Fig. 2) surgical interventions following preoperative SPECT imaging are provided.

\section{Discussion}

SPECT has been studied extensively as an aid in the diagnosis of degenerative spine disease, facetogenic pain, infections, and neoplasms in the spine. ${ }^{7}$ It has been used as a presurgical tool in other medical fields including epilepsy, and endocrine and musculoskeletal disorders. . $^{1,411}$ Few studies have looked at its role in spine surgery and most of the literature focuses on its use postoperatively. ${ }^{3}$ Our study demonstrates a potential role of preoperative SPECT imaging as a tool for surgical planning in patients with axial neck or back pain. Our results demonstrated improved clinical outcomes at 1 year postoperatively in $82.6 \%$ of patients undergoing surgery. This percentage of patients experiencing symptom relief or resolution is much greater than in previously reported studies of surgical treatment for axial back pain. Mirza et al. compared a large series of patients undergoing surgical versus nonsurgical management of chronic axial back pain, finding only
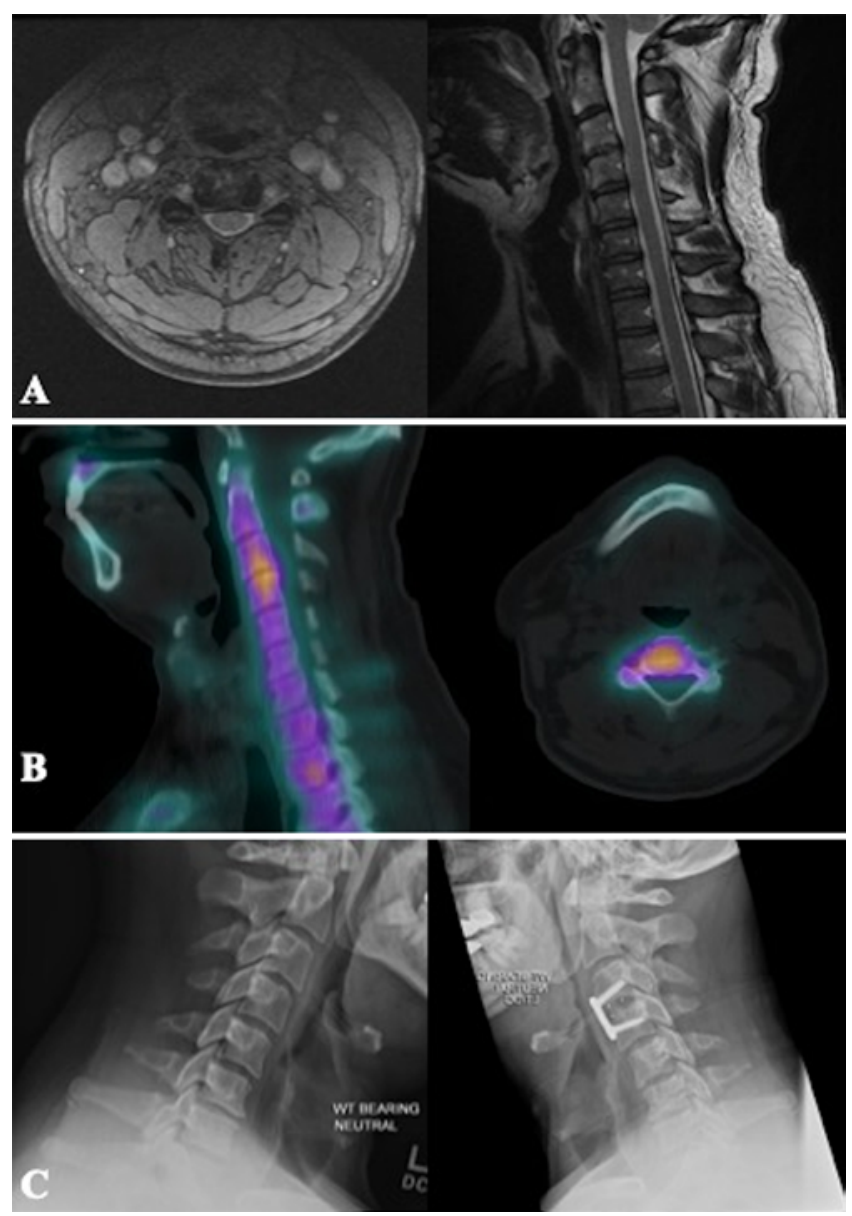

FIG. 1. Case of a 47-year-old man with a 2-year history of progressive neck pain unresponsive to conservative management. Cervical MRI (panel A) revealed mild disc degeneration at C3-4. SPECT/CT (panel B) showed hypermetabolic foci at C3-4 bodies. The patient underwent a C3-4 anterior cervical discectomy and fusion and achieved complete symptom resolution after surgery. Preoperative and postoperative cervical radiographs are shown (panel C).

a $33 \%$ success rate at 1 year in the surgical arm compared to $15 \%$ in the nonsurgical cohort. ${ }^{10}$ Furthermore, the rate of reoperation in the surgical group was $11 \%$ in this study, whereas no patients in our study required reoperation during the study period.

Our series of 23 patients is the largest published to date in which surgical treatment of axial pain following SPECT imaging is described. Only 1 study has previously examined a series of patients who underwent SPECT imaging for preoperative diagnosis of facet arthropathy. Ravindra et al. describe 7 patients with axial neck pain who were subsequently found to have upper cervical facet arthropathy on SPECT/CT and underwent posterior cervical fusion. ${ }^{13}$ Following surgical intervention, patients experienced a significant improvement on average in postoperative Neck Disability Index and visual analog scale score of $20 \%$ and 4 points, respectively, as compared to preoperative baseline. An additional study by Karam et al. used preoperative bone scintigraphy as a tool for predicting treatment response following kyphoplasty. ${ }^{5}$ The au- 

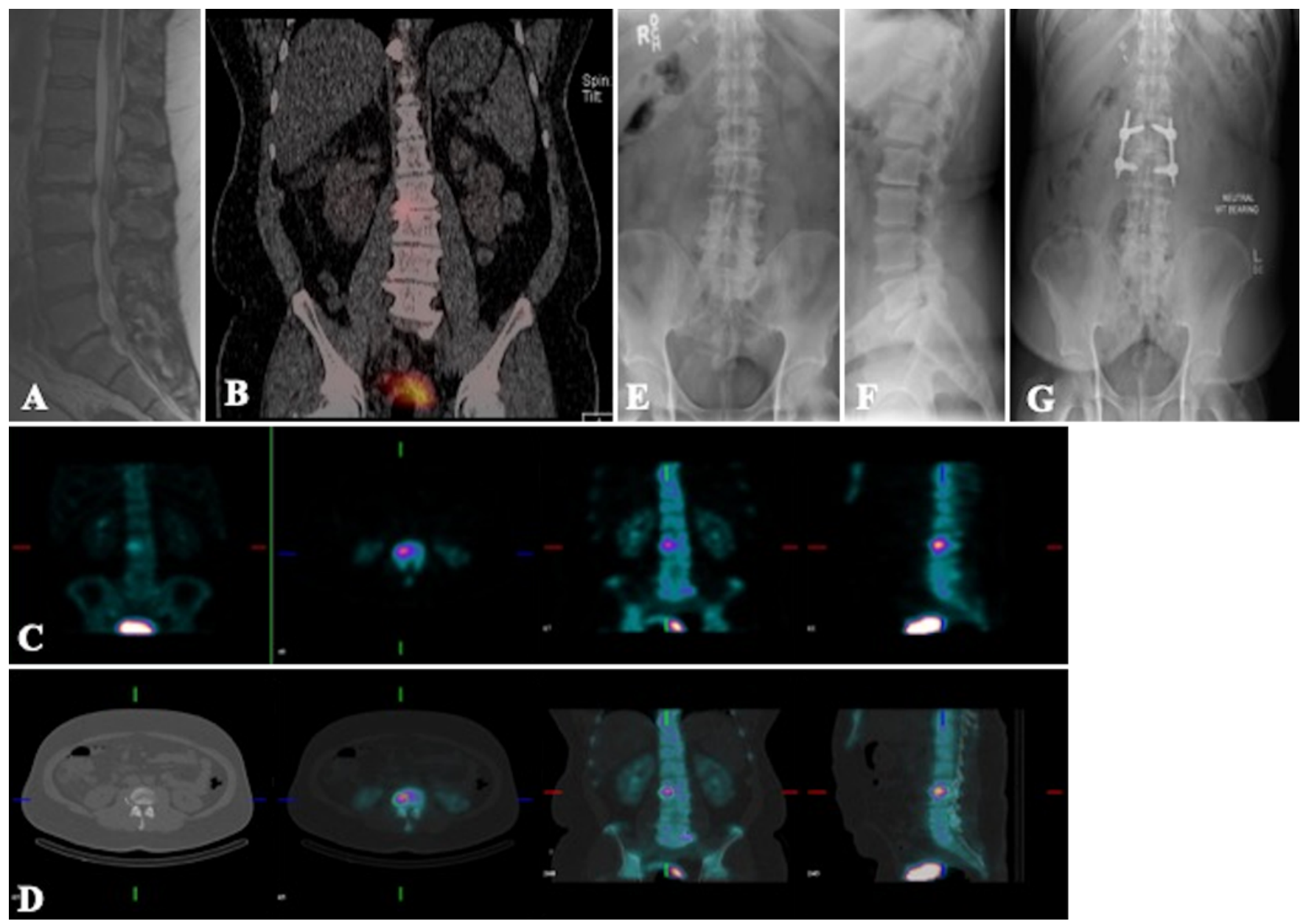

FIG. 2. Case of a 53-year-old man with a 3-year history of low-back pain that failed to respond to medical management. Lumbar MRI (panel A) demonstrated multilevel spondylosis, worse at L2-3. SPECT/CT (panels B-D) showed increased uptake at the L2-3 level on the right side. The patient underwent an awake, endoscopic L2-3 right-sided minimally invasive transforaminal lumbar interbody fusion with insertion of an intervertebral cage fixation device. Preoperative (panels $\mathbf{E}$ and $\mathbf{F}$ ) and postoperative (panel G) radiographs are shown. The patient's symptoms significantly resolved following surgery.

thors concluded that preoperative bone scanning reduced the number of vertebrae to be treated as compared to radiography alone, aided avoidance of incorrectly targeted levels, and predicted treatment response, particularly if vertebral fractures were identified.

Traditionally, facet joint injections have been used to aid in diagnosis of axial pain generators. SPECT imaging has been shown to decrease the required number of facet injections for axial back pain. ${ }^{12}$ However, a large discrepancy has been reported between hypermetabolic findings on SPECT/CT and facet joints treated with injections based on clinical symptoms; $53 \%$ of injected facets did not demonstrate any increased radiotracer uptake on SPECT imaging. ${ }^{6}$

Taken together, the use of SPECT imaging appears to enhance the decision-making process when planning surgical intervention for patients. Precise localization of active sites of degeneration with SPECT/CT may obviate the need for repeated imaging or facet injections and enable targeted surgical intervention given appropriate clinical findings.

\section{Study Limitations}

There are several important limitations to this study. First, our series is small, with just 23 patients included. However, traditional management of axial neck or back pain is nonsurgical and thus, our series is the largest one to date examining surgical outcomes. Second, a subset of patients had involvement of multiple spinal levels. Therefore, the inability to treat all degenerated levels and the progressive nature of the osteoarthritic disease may be responsible for a lack of clinical improvement in these select patients over time. Third, biases related to retrospective reviews must be taken into account, and the results of our single-surgeon, single-institution series may not be generalizable to other patient populations.

\section{Conclusions}

This is the largest series to date describing patients with axial neck and back pain who underwent preoperative SPECT imaging and subsequent surgical intervention on the affected spinal levels. Our results demonstrate that 
SPECT imaging may be a useful adjunct to guide surgical planning, resulting in substantial clinical improvement following surgery.

\section{References}

1. Akbaba G, Berker D, Isik S, Aydin Y, Ciliz D, Peksoy I, et al: A comparative study of pre-operative imaging methods in patients with primary hyperparathyroidism: ultrasonography, $99 \mathrm{mTc}$ sestamibi, single photon emission computed tomography, and magnetic resonance imaging. J Endocrinol Invest 35:359-364, 2012

2. Al-Riyami K, Vöö S, Gnanasegaran G, Pressney I, Meir A, Casey A, et al: The role of bone SPECT/CT in patients with persistent or recurrent lumbar pain following lumbar spine stabilization surgery. Eur J Nucl Med Mol Imaging 46:989-998, 2019

3. Gnanasegaran G, Paycha F, Strobel K, van der Bruggen W, Kampen WU, Kuwert T, et al: Bone SPECT/CT in postoperative spine. Semin Nucl Med 48:410-424, 2018

4. Gross CE, Barfield W, Schweizer C, Rasch H, Hirschmann MT, Hintermann B, et al: The utility of the ankle SPECT/CT scan to predict functional and clinical outcomes in supramalleolar osteotomy patients. J Orthop Res 36:2015-2021, 2018

5. Karam M, Lavelle WF, Cheney R: The role of bone scintigraphy in treatment planning, and predicting pain relief after kyphoplasty. Nucl Med Commun 29:247-253, 2008

6. Lehman VT, Murphy RC, Kaufmann TJ, Diehn FE, Murthy NS, Wald JT, et al: Frequency of discordance between facet joint activity on technetium Tc99m methylene diphosphonate SPECT/CT and selection for percutaneous treatment at a large multispecialty institution. AJNR Am J Neuroradiol 35:609-614, 2014

7. Lehman VT, Murphy RC, Maus TP: 99mTc-MDP SPECT/ $\mathrm{CT}$ of the spine and sacrum at a multispecialty institution: clinical use, findings, and impact on patient management. Nucl Med Commun 34:1097-1106, 2013

8. Makki D, Khazim R, Zaidan AA, Ravi K, Toma T: Single photon emission computerized tomography (SPECT) scanpositive facet joints and other spinal structures in a hospitalwide population with spinal pain. Spine J 10:58-62, 2010

9. Matar HE, Navalkissoor S, Berovic M, Shetty R, Garlick N, Casey AT, et al: Is hybrid imaging (SPECT/CT) a useful adjunct in the management of suspected facet joints arthropathy? Int Orthop 37:865-870, 2013
10. Mirza SK, Deyo RA, Heagerty PJ, Turner JA, Martin BI, Comstock BA: One-year outcomes of surgical versus nonsurgical treatments for discogenic back pain: a communitybased prospective cohort study. Spine J 13:1421-1433, 2013

11. Pittau F, Grouiller F, Spinelli L, Seeck M, Michel CM, Vulliemoz S: The role of functional neuroimaging in pre-surgical epilepsy evaluation. Front Neurol 5:31, 2014

12. Pneumaticos SG, Chatziioannou SN, Hipp JA, Moore WH, Esses SI: Low back pain: prediction of short-term outcome of facet joint injection with bone scintigraphy. Radiology 238:693-698, 2006

13. Ravindra VM, Mazur MD, Bisson EF, Barton C, Shah LM, Dailey AT: The usefulness of single-photon emission computed tomography in defining painful upper cervical facet arthropathy. World Neurosurg 96:390-395, 2016

14. Russo VM, Dhawan RT, Baudracco I, Dharmarajah N, Lazzarino AI, Casey AT: Hybrid bone SPECT/CT imaging in evaluation of chronic low back pain: correlation with facet joint arthropathy. World Neurosurg 107:732-738, 2017

\section{Disclosures}

Dr. Wang receives royalty payments from DePuy-Synthes Spine, Children's Hospital of Los Angeles, Springer Publishing, and Quality Medical Publishing; is a consultant for DePuy-Synthes Spine, Stryker Spine, K2M, and Spineology; is an advisory board member for Vallum; owns stock in Spinicity and Innovative Surgical Devices; and receives grants from the US Department of Defense.

\section{Author Contributions}

Conception and design: Wang. Acquisition of data: Brusko, Perez-Roman, Tapamo, Burks. Analysis and interpretation of data: Brusko, Perez-Roman, Serafini. Drafting the article: Brusko, Perez-Roman, Burks. Critically revising the article: Wang, PerezRoman, Burks, Serafini. Reviewed submitted version of manuscript: Wang, Brusko, Burks. Statistical analysis: Brusko.

\section{Correspondence}

Michael Y. Wang: Lois Pope LIFE Center, Miami, FL. mwang2@ med.miami.edu. 